\title{
¿Qué medidas para la mejora de la convivencia se están desarrollando en los centros educativos?: una perspectiva desde dentro
}

\author{
Alejandra Dobarro González, Trinidad García \\ Fernández y David Álvarez García \\ Universidad de Oviedo (España)
}

\begin{abstract}
El objetivo de este trabajo es analizar qué medidas para la mejora de la convivencia son las que el alumnado percibe que se están desarrollando más en los centros educativos. Para ello se aplicó a una muestra de 2.597 estudiantes de Educación Secundaria Obligatoria del Principado de Asturias una prueba, el Cuestionario M-ESO, con el que se evalúa la opinión del alumnado respecto a aspectos organizativos del centro y del aula relacionados con la convivencia; la resolución de conflictos en el centro; el trabajo y la metodología en el aula; la relación entre los estudiantes; y la relación entre las familias y el centro. Los resultados obtenidos muestran que de las medidas desarrolladas en su centro educativo para la mejora de la convivencia, las estrategias de difusión de normas y sanciones son las que el alumnado percibe como más habituales, seguidas de aquellas que se refieren a la educación en valores y resolución de conflictos. Contrario a lo que sería deseable, las estrategias relativas al consenso de normas son las que el alumnado de ESO percibe como menos habituales en su centro.
\end{abstract}

Palabras clave: Convivencia, violencia escolar, Educación Secundaria, consenso de normas.

What actions to improve coexistence are being developed at schools?: a inside perspective. The aim of this work was to know students` perception about different techniques to improve the school coexistence. For it, there were evaluated 2597 students of the Compulsory Secondary Education by means of the Questionnaire M-ESO. The Questionnaire $M$-ESO allows evaluate the students` opinion about organizational aspect related to coexistence, conflict resolution, teaching methodology, relationship between students and relationship between families and school. Results show that dissemination of rules and punishment are perceived by students such the most common of the actions to improve coexistence. These actions are followed in frequency by those relating to education in values and conflict resolution. Contrary to what is desirable, strategies relating to consensus rules are perceived by students such the less common in its center.

Key words: Coexistence, school violence, Secondary School, consensus rules.

Correspondencia: Alejandra Dobarro González. Departamento de Psicología. Facultad de Formación del Profesorado y Educación. Universidad de Oviedo. Calle Aniceto Sela, s/n. C.P. 33003. Oviedo (España). E-mail: dobarroalejandra@uniovi.es 
El clima de convivencia es uno de los factores que más afectan a la calidad del proceso de enseñanza-aprendizaje en los centros educativos. Las buenas relaciones entre los miembros de la comunidad educativa favorecen tanto el rendimiento académico (Moral, Sanchez, y Villarreal, 2010) como un desarrollo personal y social ajustado (Cerezo, 2008). En los últimos años, se están produciendo importantes cambios sociales en las formas de interacción y de comportamiento que se reflejan en el aula y que en ocasiones afectan a ese clima de convivencia. Estos cambios suponen todo un reto para la comunidad educativa, que trata de encontrar medidas eficaces para la gestión del aula.

Para ello, los centros educativos establecen en sus Planes de Convivencia las medidas previstas para la prevención y el tratamiento de los problemas de convivencia que puedan surgir, de acuerdo a la normativa educativa (en Asturias, Decreto 249/2007, por el que se regulan los derechos y deberes del alumnado y normas de convivencia en los centros docentes no universitarios).

Entre las posibles líneas de intervención para la mejora de la convivencia escolar, hoy en día predomina el denominado modelo integrado (Torrego, 2001, 2006), según el cual el centro debe disponer de un sistema de normas y sanciones claro y conocido por toda la comunidad educativa, pero también de mecanismos para la resolución constructiva de conflictos, complementarios o alternativos al sistema normativo existente (Barriocanal, 2001; Crawford y Bodine, 1996; Torrego y Moreno, 2003; Watkins y Wagner, 1991). En distintas disposiciones normativas (dependiendo de la Comunidad Autónoma) se concreta que cada centro incorporará en su Reglamento de Régimen Interior un plan destinado a mejorar la convivencia escolar, y en cuya elaboración han de participar todos los sectores de la Comunidad Educativa. En este Plan de Convivencia se establecerán unas normas generales para todo el centro y unas específicas para las distintas aulas, normas encaminadas a hacer efectivo el respeto de los derechos y libertades fundamentales y la igualdad de trato y no discriminación, de acuerdo a los principios de un sistema democrático.

Investigadores y docentes (Barriocanal, 2001; Torrego y Moreno, 2003; Watkins y Wagner, 1991) vienen insistiendo en la conveniencia no sólo de difundir las normas de convivencia en el aula entre el alumnado, sino que estas normas de aula sean elaboradas de manera consensuada con la participación del alumnado. La participación democrática del alumnado en la elaboración de un sistema de normas claras de convivencia para el aula y de las consecuencias de su violación, hace que estos las conozcan, se impliquen en el buen funcionamiento del aula y las acepten con mayor facilidad. Explicitar -dar formalidad- unas normas que suelen ser implícitas -informalesda lugar a que el alumnado sepa a qué atenerse y no vean las sanciones como algo que el profesorado aplica con arbitrariedad. Además, si se elaboran normas -y correccionestambién para el profesorado, los alumnos pueden aceptar mejor el sistema normativo e implicarse en su cumplimiento, al ver que el compromiso por el buen clima del aula es 
compartido, recíproco. Una de las finalidades del Sistema Educativo, recogida en la Ley Orgánica 2/2006 de Educación, es la educación de todo el alumnado en la prevención y resolución pacífica de conflictos, de manera que se contribuya a generar un buen clima de convivencia, tan necesario para alcanzar el resto de objetivos educativos. De hecho, la Educación en Resolución de Conflictos es una de las líneas de intervención para la mejora de la convivencia que está recibiendo una mayor atención por parte de investigadores y educadores en la actualidad. Aglutina todo un variado conjunto de experiencias que tienen en común la práctica o formación de al menos uno de los procesos que permiten afrontar constructivamente conflictos -negociación, mediación y consenso en grupo- así como los principios, habilidades y actitudes necesarios para ponerlos en marcha (Crawford y Bodine, 1996). Estas prácticas pueden ir, desde el profesor o la profesora que aisladamente decide formar a su alumnado en estos contenidos, hasta un plan global del centro en el que se combine la formación -ya no sólo del alumnado, sino también del profesorado y de las familias- con prácticas para la gestión constructiva de conflictos como el servicio de mediación, la red de alumnos ayudantes, la asamblea de aula o el consenso de normas.

El objetivo de este trabajo es evaluar, desde la perspectiva del alumnado, qué medidas para la mejora de la convivencia de las anteriormente expuestas (educación en valores y resolución de conflictos, difusión de normas y sanciones, y consenso de normas de aula) se están desarrollando más en los centros educativos.

\section{MÉTODO}

\section{Participantes}

La muestra está compuesta por un total de 2.597 estudiantes de $1^{\circ}$ a $4^{\circ}$ de Educación Secundaria Obligatoria, pertenecientes a 18 centros en los que se cursan esos estudios. La mayoría de ellos cursan sus estudios en centros públicos $(70.97 \%$, frente al $29.03 \%$ que lo hace en centros concertados). Estos estudiantes se dividen de forma proporcional en los cuatro niveles de la etapa, así el $28.61 \%$ cursa $1^{\circ}$ de ESO, el $25.64 \%$ lo hace en $2^{\circ} \mathrm{ESO}$, el $22.33 \%$ se encuentra cursando $3^{\circ}$ ESO y el $23.41 \%$ está en $4^{\circ}$ curso de ESO. Respecto a la variable género, el $48.16 \%$ son alumnas y el $49.26 \%$ son alumnos.

Tabla 1. Características de la muestra utilizada en el estudio

\begin{tabular}{|c|c|c|c|c|c|c|c|c|c|}
\hline & \multicolumn{4}{|c|}{ Públicos } & \multicolumn{4}{|c|}{ Concertados } & \multirow{2}{*}{ Total } \\
\hline & Alumnos & Alumnas & $\mathrm{NC}$ & Total & Alumnos & Alumnas & $\mathrm{NC}$ & Total & \\
\hline $1^{\circ} \mathrm{ESO}$ & 271 & 236 & 18 & 525 & 103 & 110 & 5 & 218 & 743 \\
\hline $2^{\circ} \mathrm{ESO}$ & 212 & 223 & 23 & 458 & 114 & 89 & 5 & 208 & 666 \\
\hline $3^{\circ} \mathrm{ESO}$ & 208 & 195 & 12 & 415 & 79 & 85 & 1 & 165 & 580 \\
\hline $4^{\circ} \mathrm{ESO}$ & 204 & 226 & 15 & 445 & 79 & 81 & 3 & 163 & 608 \\
\hline Total ESO & 895 & 880 & 68 & 1843 & 375 & 365 & 14 & 754 & 2597 \\
\hline
\end{tabular}

$\mathrm{NC}=$ No contesta; $\mathrm{ESO}=$ Educación Secundaria Obligatoria 


\section{Instrumento de evaluación}

Para realizar este estudio se ha utilizado el Cuestionario M-ESO: Cuestionario para la evaluación de la presencia de medidas para la mejora de la convivencia escolar percibida por el alumnado de Educación Secundaria Obligatoria, cuya validez y fiabilidad ya habían sido analizadas por este equipo de investigación (trabajo aún sin publicar).

El Cuestionario $M$ adopta la forma de una escala de 22 ítems tipo Likert con 4 opciones de respuesta ( $1=$ Totalmente en desacuerdo; 2=En desacuerdo; 3=De acuerdo; 4=Totalmente de acuerdo), en la que el evaluado debe indicar en qué medida está o no de acuerdo con cada afirmación, referida a la existencia de diferentes tipos de medidas para la mejora de la convivencia escolar. Tomado en su conjunto el cuestionario presenta un índice de fiabilidad moderado-alto $(\alpha=.899)$. Este índice, si bien es algo menor en cada uno de los de tres factores que conforman su estructura, continúa siendo aceptable: Difusión de normas y sanciones $(\alpha=.849)$, Consenso de normas de aula $(\alpha=.733)$ y Educación en valores y resolución de conflictos $(\alpha=.784)$.

\section{Procedimiento}

Se realizó un muestreo por conglomerados de entre la población de centros de enseñanza secundaria obligatoria del Principado de Asturias sostenidos con fondos públicos -públicos y concertados-. Se formaron categorías a partir del censo de centros del curso 2009/2010 (un total de 147 centros, con 30.102 estudiantes de ESO matriculados) y del área CPR a la que pertenece cada centro. De este modo, se obtuvieron 7 categorías de centros, eligiéndose en cada una de ellas, y al azar, un número de centros proporcional al tamaño del estrato dentro de la población. Finalmente se evaluaron 18 centros de Educación Secundaria con un total de 2.597 estudiantes de ESO.

Una vez seleccionados los centros, se solicitó permiso a sus respectivos equipos directivos para realizar la investigación. Cada equipo directivo fue informado de los objetivos y procedimientos del estudio, de su carácter voluntario y anónimo, y del tratamiento confidencial de los resultados. El cuestionario fue aplicado en todos los centros a mediados del tercer trimestre del curso 2009/2010. Antes de contestar al cuestionario, los estudiantes también fueron informados del objetivo del estudio y de su carácter anónimo, confidencial y voluntario. La prueba fue aplicada por el equipo investigador a todos los grupos de cada uno de los centros, en horario lectivo.

\section{RESULTADOS}

De los tres tipos de medidas para la prevención de la violencia escolar que permite evaluar el Cuestionario M-ESO, el más habitual en opinión del alumnado de Educación Secundaria Obligatoria es la Difusión de las Normas y Sanciones (Tabla 2). 
De hecho, tal como se observa en la tabla 3, los siete enunciados del Cuestionario M-ESO que han obtenido mayor puntuación forman parte de ese factor. Concretamente, la medida que más considera el alumnado de ESO que existe en su centro es que sus familias hayan sido informadas de las normas del centro (ítem 1), seguido de la existencia de unas normas claras de convivencia en el aula (ítem 6) y del conocimiento por parte del alumnado de las sanciones que se aplicarán en el caso de no respetar las normas (ítem 2).

Tabla 3. Descriptivos de los ítems del Cuestionario M-ESO, por orden de frecuencia $(N=2.597)$

\begin{tabular}{|c|c|c|c|c|c|c|c|c|c|}
\hline \multirow{2}{*}{\multicolumn{2}{|c|}{ Ítem }} & \multirow{2}{*}{ Enunciado } & \multirow{2}{*}{ Media } & \multirow{2}{*}{$D T$} & \multicolumn{5}{|c|}{ Escala $(\%)$} \\
\hline & & & & & 1 & 2 & 3 & 4 & $\mathrm{NC}$ \\
\hline $1^{\circ}$ & 1 & Las familias han sido informadas de las normas del centro. & 3.24 & 0.88 & 6.0 & 11.1 & 35.6 & 47.2 & 0.0 \\
\hline $2^{\circ}$ & 6 & Existen unas normas claras de convivencia en el aula. & 3.16 & 0.81 & 4.5 & 12.8 & 44.1 & 38.2 & 0.4 \\
\hline $3^{\circ}$ & 2 & $\begin{array}{l}\text { El alumnado conoce las sanciones a aplicar en caso de no } \\
\text { respetar las normas de convivencia en el aula. }\end{array}$ & 3.06 & 0.86 & 6.2 & 15.7 & 43.3 & 34.5 & 0.3 \\
\hline $4^{\circ}$ & 7 & El alumnado conoce las normas de convivencia en el aula. & 3.05 & 0.82 & 5.1 & 15.7 & 47.5 & 31.2 & 0.5 \\
\hline $5^{\circ}$ & 5 & $\begin{array}{l}\text { Las normas de convivencia en el aula se han plasmado por } \\
\text { escrito. }\end{array}$ & 3.01 & 0.98 & 10.2 & 16.6 & 34.3 & 38.3 & 0.6 \\
\hline $6^{\circ}$ & 4 & $\begin{array}{l}\text { Las sanciones por incumplimiento de las normas del centro } \\
\text { se han dado a conocer a las familias. }\end{array}$ & 2.94 & 0.91 & 8.4 & 19.5 & 41.3 & 30.4 & 0.4 \\
\hline $7^{\circ}$ & 20 & $\begin{array}{l}\text { Las sanciones por el incumplimiento de las normas de clase } \\
\text { se dan a conocer a las familias. }\end{array}$ & 2.94 & 0.89 & 8.0 & 19.1 & 43.0 & 29.1 & 0.7 \\
\hline $8^{\circ}$ & 18 & Se fomenta el respeto por la diversidad de las personas. & 2.90 & 0.79 & 6.6 & 17.3 & 55.1 & 20.3 & 0.8 \\
\hline $9^{\circ}$ & 15 & $\begin{array}{l}\text { Valores, como la convivencia. se fomentan en clase o } \\
\text { tutoría. }\end{array}$ & 2.89 & 0.87 & 8.6 & 18.6 & 48.2 & 24.3 & 0.3 \\
\hline $10^{\circ}$ & 3 & $\begin{array}{l}\text { Ante conflictos entre alumnado, el profesorado se esfuerza } \\
\text { en ayudarles a que lo resuelvan, mediando entre ellos. }\end{array}$ & 2.80 & 0.88 & 8.5 & 25.1 & 43.2 & 22.6 & 0.6 \\
\hline $11^{\circ}$ & 11 & $\begin{array}{l}\text { El profesorado realiza actividades que favorecen la } \\
\text { participación y el conocimiento entre el alumnado. }\end{array}$ & 2.80 & 0.84 & 8.5 & 21.3 & 51.1 & 18.5 & 0.6 \\
\hline $12^{\circ}$ & 21 & $\begin{array}{l}\text { Cada cierto tiempo se celebran en tutoría reuniones en las } \\
\text { que se tratan temas relacionados con el grupo. }\end{array}$ & 2.80 & 0.93 & 11.4 & 20.9 & 43.2 & 24.0 & 0.5 \\
\hline $13^{\circ}$ & 9 & Las familias han sido informadas de las normas de la clase. & 2.77 & 0.98 & 13.3 & 22.3 & 38.1 & 25.7 & 0.7 \\
\hline $14^{\circ}$ & 16 & $\begin{array}{l}\text { El alumnado conoce las normas de convivencia del centro, } \\
\text { contenidas en el Reglamento de Régimen Interior. }\end{array}$ & 2.74 & 0.95 & 12.8 & 23.1 & 41.2 & 22.4 & 0.5 \\
\hline $15^{\circ}$ & 17 & $\begin{array}{l}\text { Se enseñan estrategias para resolver los conflictos de } \\
\text { manera no violenta, a través del dialogo y el respeto. }\end{array}$ & 2.70 & 0.89 & 11.4 & 25.2 & 44.8 & 18.1 & 0.5 \\
\hline $16^{\circ}$ & 13 & $\begin{array}{l}\text { Los problemas diarios que afectan a la clase se tratan de } \\
\text { solucionar entre todos, de manera dialogada. }\end{array}$ & 2.67 & 0.90 & 12.0 & 26.0 & 44.0 & 17.4 & 0.6 \\
\hline $17^{\circ}$ & 22 & $\begin{array}{l}\text { El alumnado conoce las sanciones incluidas en el RRI, por } \\
\text { incumplimiento de las normas de centro. }\end{array}$ & 2.64 & 0.94 & 14.0 & 26.4 & 40.0 & 19.1 & 0.5 \\
\hline $18^{\circ}$ & 19 & $\begin{array}{l}\text { El profesorado aplica las normas y sanciones de forma } \\
\text { justa. }\end{array}$ & 2.59 & 0.89 & 12.2 & 31.6 & 40.6 & 15.1 & 0.5 \\
\hline $19^{\circ}$ & 14 & $\begin{array}{l}\text { La clase, junto con el profesorado, determina las } \\
\text { consecuencias en caso de incumplimiento de las normas. }\end{array}$ & 2.42 & 0.92 & 18.4 & 31.8 & 37.7 & 11.4 & 0.7 \\
\hline $20^{\circ}$ & 12 & $\begin{array}{l}\text { Se valora la opinión del alumnado para cambiar ciertas } \\
\text { normas de clase. }\end{array}$ & 2.17 & 0.92 & 27.1 & 36.5 & 27.6 & 8.4 & 0.5 \\
\hline $21^{\circ}$ & 10 & $\begin{array}{l}\text { Las normas de convivencia en el aula han sido elaboradas } \\
\text { con la participación del alumnado. }\end{array}$ & 2.10 & 0.98 & 33.5 & 32.3 & 23.3 & 10.1 & 0.7 \\
\hline $22^{\circ}$ & 8 & $\begin{array}{l}\text { Cada cierto tiempo profesorado y alumnado revisan las } \\
\text { normas y su grado de cumplimiento. }\end{array}$ & 1.97 & 0.94 & 37.9 & 33.7 & 20.5 & 7.3 & 0.6 \\
\hline
\end{tabular}

$D T=$ Desviación Típica; 1=Totalmente en desacuerdo; 2=En desacuerdo; 3=De acuerdo; 4=Totalmente de acuerdo; $\mathrm{NC}=$ No contesta 
El segundo tipo de medidas más habituales, según el alumnado de ESO, se refieren a la Educación en Valores y Resolución de Conflictos (Tabla 2). De hecho, en el análisis por ítems, tras los siete enunciados referidos a Difusión de las Normas y Sanciones arriba citados, aparecen cuatro de los siete enunciados referidos a Educación en Valores y Resolución de Conflictos (Tabla 3). En los puestos $8^{\circ}$ a $12^{\circ}$ aparecen los ítems "Se fomenta el respeto por la diversidad de las personas" (ítem 18); "Valores, como la convivencia, se fomentan en clase o tutoría" (ítem 15); "Cuando hay conflictos entre el alumnado, el profesorado se esfuerza en ayudarles a que lo resuelvan, mediando entre ellos" (ítem 3); y "El profesorado realiza actividades que favorecen el conocimiento y la participación del alumnado" (ítem 11).

El tipo de medida para la mejora de la convivencia que el alumnado de Educación Secundaria Obligatoria considera menos habitual es el Consenso de Normas de Aula (Tabla 2). Los ítems que conforman este factor aparecen en los últimos lugares de la tabla 3, pues presentan las medias más bajas. Estos se refieren a medidas como las reflejadas en el ítem 8 ("Cada cierto tiempo profesorado y alumnado revisan las normas y su grado de cumplimiento"), en el ítem 10 ("Las normas de convivencia en el aula han sido elaboradas con la participación del alumnado") o en el ítem 12 ("Se valora la opinión del alumnado para cambiar ciertas normas de clase").

\section{CONCLUSIONES}

En nuestro país no existen demasiados estudios que evalúen desde la perspectiva del alumnado qué medidas, de las que sabemos mejoran el clima de convivencia escolar, se están desarrollando en los centros educativos. De tal modo, los resultados que aquí se presentan supones una aportación al estudio de la convivencia escolar. Este estudio, además, utiliza como instrumento una prueba específica, breve, de fácil aplicación y corrección, y útil, pues ha permitido a este equipo investigador no sólo describir la realidad en las aulas, desde la perspectiva del alumnado, en cuanto a las variables que evalúa, sino también analizar hasta qué punto esas variables permiten predecir otras variables relevantes, como el grado de aparición de violencia escolar (Álvarez-García, Dobarro, Rodríguez, Núñez y Álvarez, 2011 trabajo enviado para su publicación). Al comparar estos resultados con los obtenidos en otro estudio previo realizado con estudiantes de Educación Primaria (Álvarez-García, Núñez, Dobarro y Rodríguez, 2012), se comprueba la existencia de una tendencia general a que el alumnado considere cada vez menos habituales, a medida que avanza su nivel educativo, la puesta en marcha de las medidas para la mejora de la convivencia. Por otro lado, se aprecia una evolución, de tercer ciclo de Educación Primaria a Educación Secundaria Obligatoria, hacia estrategias basadas en la difusión de normas y sanciones, en detrimento de estrategias basadas en la educación en resolución de conflictos, que 
predominan más en tercer ciclo de Primaria. Tanto en tercer ciclo de Primaria como en Educación Secundaria Obligatoria, el tipo de medidas menos habituales, según el alumnado, son las relacionadas con la gestión democrática de normas y sanciones.

Los resultados muestran que estrategias basadas en la Educación en Valores y Resolución de Conflictos y en el Consenso de Normas de Aula son las que el alumnado percibe como menos habituales en sus centros educativos. Esto parece contrario a lo deseable y lo que cabría esperar, pues son conocidos los beneficios que presentan este tipo de medidas sobre el clima de convivencia escolar, y por tanto sobre otras variables relacionadas como el rendimiento académico, el nivel de violencia escolar o el desarrollo personal (Álvarez-García et al., 2011 trabajo enviado para su publicación). Estos datos deberían llevar a investigadores y docentes a la búsqueda de intervenciones más afinadas y concretas, a la vez que más frecuentes y visibles, con el fin de prevenir distintos tipos de violencia escolar y mejorar el clima de convivencia.

\section{REFERENCIAS}

Álvarez-García, D., Dobarro, A., Rodríguez, C., Núñez, J.C. y Álvarez, L. (2011). El consenso de normas de aula como predictor de bajos niveles de violencia escolar. Trabajo enviado para su publicación.

Alvarez-García, D., Núñez, J.C., Dobarro, A. y Rodríguez, C. (2012). Propiedades psicométricas del Cuestionario de Medidas para la Mejora de la Convivencia en Educación Primaria (Cuestionario M-EP). Revista Electrónica de Metodología Aplicada, 17(2), 1-17.

Barriocanal, L.A. (2001). Implicando al alumnado en el establecimiento de normas de clase: normas consensuadas, normas aceptadas. En I. Fernández (Ed.), Guía para la convivencia en el aula (pp. 73-99). Barcelona: CISS PRAXIS.

Cerezo, F. (2008). Acoso escolar. Efectos del bullying. Boletín de la Sociedad de Pediatría de Asturias, Cantabria, Castilla y León, 48, 353-358.

Crawford, D. y Bodine, R. (1996). Conflict resolution education. A guide to implementing programs in schools, youth-serving organizations, and community and juvenile justice settings. Washington, DC: U.S. Department of Justice, U.S. Department of Education.

Moral, J.C., Sánchez, J.C. y Villarreal, M.E. (2010). Desarrollo de una Escala Multidimensional Breve de Ajuste Escolar. R.E.M.A. Revista Electrónica de Metodología Aplicada, 15(1), $1-11$.

Torrego, J.C. (2001). Nuevos enfoques de actuación ante el conflicto y los problemas de la convivencia escolar. En I. Fernández (Ed.), Guía para la convivencia en el aula (pp. 171-179). Barcelona: CISSPraxis.

Torrego, J.C. (2006). Modelo integrado de mejora de la convivencia. Estrategias de mediación y tratamiento de conflictos. Barcelona: Graó.

Torrego, J.C. y Moreno, J.M. (2003). Convivencia y disciplina en la escuela. El aprendizaje de la democracia. Madrid: Alianza.

Watkins, C. y Wagner, P. (1991). La disciplina escolar. Propuesta de trabajo en el marco global del centro. Barcelona: Paidós-MEC.

Recibido: 2 de marzo de 2013

Recepción Modificaciones: 8 de marzo de 2013

Aceptado: 11 de septiembre de 2013 